\title{
A User-Oriented Design Knowledge Reuse Model
}

\author{
Jia Hao, ${ }^{1,2}$ Yan Yan, ${ }^{1}$ Guoxin Wang, ${ }^{1}$ Lin Gong, ${ }^{1}$ and Jianjun Lin ${ }^{3}$ \\ ${ }^{1}$ School of Mechanical Engineering, Beijing Institute of Technology, Beijing 100081, China \\ ${ }^{2}$ Department of Mechanical Engineering, University of Michigan, Ann Arbor, MI 48105, USA \\ ${ }^{3}$ China North Vehicle Research Institute, Beijing 100071, China \\ Correspondence should be addressed to Jia Hao; haojia632@gmail.com
}

Received 5 June 2013; Accepted 25 July 2013

Academic Editors: T.-M. Choi and A. Gomez

Copyright (C) 2013 Jia Hao et al. This is an open access article distributed under the Creative Commons Attribution License, which permits unrestricted use, distribution, and reproduction in any medium, provided the original work is properly cited.

\begin{abstract}
To promote the level of reusing the accumulated design knowledge in the company, we intend to develop a knowledge reuse model in this paper. First, the design knowledge is discussed, and a unified design knowledge model is proposed. Following that, we identify three design knowledge reuse patterns which contribute to the understanding of how designers reuse knowledge during the design process. Based on that, we propose a design knowledge reuse model. This model can provide design knowledge according to the design knowledge reuse patterns. The model also provides the ability to update itself according to the captured actions of the knowledge users. At last, the method is validated with a research cooperator on the hydropneumatic spring component.
\end{abstract}

\section{Introduction}

A large number of researchers appeal that the active utilization of a company's knowledge is vital to maintaining competitive advantage in today's knowledge-based economy, particularly within design and manufacturing environments [1-3]. The reuse of existing design knowledge benefits both new design and redesign. For a new design, the designer has great freedom in choosing the structure or deciding the value of the parameters, while, for a redesign process, a designer may concentrate his/her attention on the design defects and problems occurring in the existing design and the customers' new needs of the existing product. However, the levels of knowledge reuse in manufacturing companies are significantly low [4].

To promote the level of design knowledge reuse, many research works are carried out. At the beginning, researches on design knowledge management focus on content search by matching keyword and file name. However, many shortcomings are realized by researchers as stated by [5]. The most important drawback is that design knowledge is often incomplete or is not adequately defined at a detailed level for current information search methods. Consistent with that an integrated design knowledge model should benefit the knowledge reuse, many design knowledge reuse models are proposed and studied, like process-based knowledge reuse model [6], functions based knowledge reuse [1], ontological knowledge reuse model [7], and CAD/CAE based knowledge reuse which is widely known as knowledge-based engineering (KBE). Many techniques from artificial intelligence (AI), like case-based reasoning (CBR) and Bayesian network, are adopted to solve the problem of design knowledge reuse $[2,4]$. In recent research literature, many researchers point out that further effort is required to understand the needs of knowledge user and producers in order that appropriate methods can be applied $[4,6,8]$.

The aim of this paper is to identify the knowledge reuse patterns of the knowledge user during the product design process and to build a design knowledge reuse model in accordance with the knowledge reuse patterns. The remaining content of this paper is organized as follows. Section 2 reviews the literatures related design knowledge reuse model, method, and applications. Section 3 investigates design knowledge and proposes a unified design knowledge model. Section 4 identifies four knowledge reuse patterns of the designers to explain how designers reuse design knowledge during the product design process. Section 5 proposes a design knowledge reuse model to serve the knowledge reuse demands. Section 6 shows a case study in which the 
knowledge reuse model of the hydropneumatic spring is constructed and demonstrated. Section 7 summarizes the work and points out the future work.

\section{Related Works}

To address the knowledge reuse problem, many methods are proposed like CAD/CAE based method, function based method, process based-method, and ontology based method.

The CAD/CAE based knowledge reuse is to embed design knowledge in the product model so that the knowledge can be reused and generate new product model. Knowledgebased engineering (KBE) technology is a prevalent method to achieve such kind of knowledge reuse. In recent years, $\mathrm{KBE}$ becomes a research hotspot. Verhagen et al. [9] and Rocca [10] publish two literature reviews, respectively, which offer a broad technological review of $\mathrm{KBE}$ to increase the level of understanding of its technology fundamentals. Rocca [10] argues that the method should facilitate parametric modeling of complex products, investigation of multidisciplinary performances, and optimization in a reliable and time efficient manner. In a special issue of the journal of Advanced Engineering Informatics, there are six literatures which demonstrate the recent methodologies and techniques to build knowledge-based engineering systems [11-16].

Function based method is another favorite approach which organizes design knowledge by function decomposition. Jia et al. [17] proposed a construction method for the initialized design knowledge model. This method adopts functional feature partition via mapping function to the structural feature from top to bottom of the partition tree. And the object-oriented based modularized design knowledge representation is adopted into the method. Tama and Reidsema [18] developed a methodology to identify generic product knowledge and information in a virtual collaboration environment. This method levers the function behavior structure (FBS) to be the knowledge classification approach. The author represents the knowledge classification through ontology. This method is relatively simple but provides flexibility across the various different types of product. The above-mentioned two approaches still adopt the original oldfashioned function decomposition method as with the previous research work. Ammar et al. [1] provided a new thinking of managing engineering knowledge based on function. They mark each item of design knowledge by the elementary function, expressed in the form of a verb plus one or two complement(s). Therefore, the knowledge user can retrieve knowledge by simply sending the function they need; the knowledge base will return all the information related to the function. Meanwhile, the authors realize that the knowledge base must use a standard and restricted vocabulary to ensure both uniqueness and ease of structuring. Considering that, the verb base and the complements base are constructed separately.

After the research work of Baxter et al. [6], processbased knowledge reuse attracts more research attention. Their work comprises a process model constructed using design roadmap framework for indexing and retrieval of information, a product model combining product data and ontology, and a process logic engine which interacts with the process model and serves as a trigger for the use of product data. Based on the process-based knowledge reuse model, Baxter et al. [8] propose a new framework to manage product requirements as part of an integrated product knowledge reuse method. The requirements management element is to support gathering, analyzing, selecting, documenting, verifying, and managing the requirement, while the processbased knowledge reuse model combines design process, task knowledge, and product knowledge to enable the reuse of design knowledge. And then Baxter et al. [19] developed a product service system (PSS) knowledge reuse framework, based on the findings from the analysis of design, manufacturing, and service knowledge in three manufacturing organizations. Three components are included in the framework including a design process-based model, manufacturing capability knowledge, and service knowledge. The design process-based model is used to associate design knowledge with design tasks. Similar to Baxter's research work, Feng and Cui [20] proposed a process template centered on design data integrated management architecture. A new digital specialized design component is constructed for the integration of design resources. And the architecture is designed which can provide an operational environment for the merger of engineering management and project management; it also implements design data and component reuse across different design projects. Similar structures can also be found in some process-oriented decision support systems [21-24].

Ontology represents knowledge as a set of concepts within a domain and the relationships between pairs of concepts. Numerous researchers represent design knowledge model by ontology. In his thesis, Kumar [25] developed an ontology of engineering design activities, called the Design Activity Ontology (DAO). The DAO is an extension and refinement of the work of Sim and Duffy [26]. The final DAO he built consists of 82 information flows and 25 activities. The ontology provides a formalized and structured vocabulary of design activities for consistency and exchange of design process models; therefore this ontology may benefit the process-based knowledge reuse model by making the model more consistent and explicit. Liu et al. [27] proposed an ontology based knowledge modeling approach and reuse strategy towards redesign process. They divide redesign activities into parametric redesign and structural redesign, and corresponding design knowledge will be provided when doing redesign activity. The thinking is interesting; however, the authors do not show the detail of the framework. So the procedure to build the ontology and the mapping between design knowledge are unclear. Yu [28] proposed an ontology based knowledge management approach. The author constructs an ontology of product structure and the related parameters which act as the design knowledge model. What is more is that a metamodel is also built up to constrain the domain ontology. However, the author does not say any word about how to build the linkage between the ontology model and accumulated design knowledge.

Technologies from AI, like case base reasoning (CBR), act as the role of design knowledge processing method within the context of design knowledge reuse. K. Kim and 
TABLE 1: Design knowledge in product design process [32].

\begin{tabular}{lcccc}
\hline Pictorial & Symbolic & Linguistic & Virtual & Algorithmic \\
\hline & Decision tables & Customer requirements & CAD models & Mathematical equations \\
Sketches & Production rules & Design rules, constraints & CAE simulations & Parameterizations \\
Detailed drawings & Flow charts & Analogies & Virtual reality & Constraint solvers \\
Charts & FMEA diagram & Customer feedback & Simulations & Computer algorithms \\
Photographs & Assembly tree & Verbal communication & Virtual prototypes & Design/operational \\
CAD model views & Fishbone diagrams & Concept & Animations & procedures \\
& ontologies & Solutions & Multimedia & \\
\hline
\end{tabular}

Y. S. Kim [4] presented a causal design knowledge representation method. A mathematical comparison of the procedural knowledge and the causal knowledge is conducted, and they find that the procedural knowledge model is inferior to the casual knowledge model because causal knowledge model can represent more knowledge than procedural knowledge model. And then Kim represents the casual knowledge model by a network in his 2012 research work. To integrate the casual knowledge network with next-generation CAD systems, he proposes a degree of casual representation- (DCR-) based knowledge evaluation method to evaluate contained knowledge in the network. Song and Jiang [29] studied a proactive search which enabled context-sensitive knowledge supply method. Technologies of AI and nature language processing (NLP) are adopted to extract the engineering knowledge requirement. With the help of this method, knowledge users have no need to suspend their work and conceive the keywords to search with, undergo extensive reading, and eventually recover the previous work.

Through the literature analysis, we found that the methods tend to combine together to achieve new knowledge reuse model. For example, Rocca [10] believes that KBE originated in AI; Tama and Reidsema [18] represent their FBS-based knowledge model by ontology; Kumar [25] develops the Design Activity Ontology (DAO) which gives birth to the possibility of representing process model by ontology; AI technology is not only used in knowledge processing but also applied in knowledge representation. Bryson et al. [7] develop a more comprehensive method aiming at making design knowledge reused not only horizontally (passing knowledge between current employees) but also vertically (passing knowledge between generations of employees). Although the author did not put forward detailed and achievable techniques of the method, it indeed includes process modeling, ontology technology, and that AI technology to fulfill design knowledge reuse.

At the same time, we also found the above design knowledge reuse models ignore the behaviors and needs of the knowledge users, and further effort is required to understand the needs of knowledge users and producers in order that appropriate methods can be applied $[3,4,6,8$, 30]. Therefore, the driven force of the research work is to identify knowledge reuse pattern of the knowledge user and to develop a user-centered knowledge reuse model based on the existing knowledge reuse models.

\section{Product Design Knowledge}

Successfully capturing design knowledge, effectively representing it, and easily accessing it are crucial to improve the design process. However, when it refers to the term of knowledge, no researcher can give the definition that is accepted by all researchers coming from different research areas. Even in the context of engineering design there are various meanings of the term. Many papers with "knowledge" in the title (particularly within engineering) do not attempt to address the issue of what knowledge is; they simply provide methods and tools for the business of managing it [6].

The types of knowledge carrier give an opportunity to classify design knowledge according to [31]. They believe there are five classes of design knowledge carrier including pictorial, symbolic, linguistic, virtual and algorithm. Chandrasegaran et al. [32] organized this classification by a table with some examples for each type (Table 1).

To manage the heterogeneous design knowledge, a generic knowledge model should be developed. We develop a unified design knowledge model in this paper. As shown in Figure 1, the model consists of five parts that are bounded together wrapping design knowledge with a unified cover.

The knowledge content is the body of design knowledge, and the specialized content is stored or linked here. In this paper, it is very noteworthy that we consider that all design knowledge contents should be processed and interpreted by their corresponding tools (e.g., Pro/E or CATIA can be the interpreter of CAD models), and it is unwise and impossible to develop a universal tool to interpret all kinds of design knowledge. Therefore, the interpreter corresponding to the specialized content should also be set up in this part.

The knowledge header is similar to the abstract of a professional paper which includes the primary information of design knowledge. This part adopts six simple slots by which designer can make the preliminary judgment whether the design knowledge is required or not.

The knowledge lifecycle tracks the path of design knowledge from entering into repository to getting out. The path is the guarantee that the design knowledge is under control, and the content is credible. This part is necessary and important because the design knowledge must adapt to the continuously changing situation of the organization.

The knowledge action records designers' activities that are related to current design knowledge. Six types of activities are 


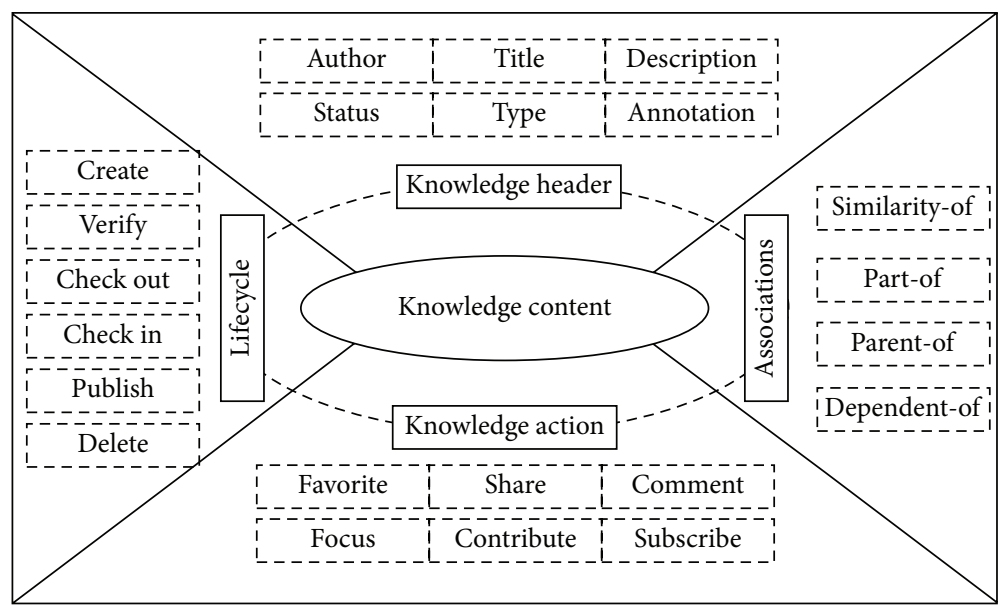

FIGURE 1: Unified design knowledge model.

TABLE 2: Description of design knowledge associations.

\begin{tabular}{|c|c|c|c|c|}
\hline Relationship & Description & Sign & Features & Sample \\
\hline Similarity-of & $\begin{array}{l}\text { Implies there is similar content in } \\
\text { two-design knowledge. This relation can } \\
\text { be built by automatic semantic analysis or } \\
\text { by domain knowledge expert }\end{array}$ & $\cong$ & $\begin{array}{l}\text { Self-inverse } \\
\text { transitivity }\end{array}$ & $\begin{array}{l}\mathrm{A} \cong \mathrm{B} \text { means the content in knowledge } \mathrm{A} \\
\text { is similar to the content in knowledge } \mathrm{B} \text {. } \\
\text { And it can be inferred the opposite is also } \\
\text { true }\end{array}$ \\
\hline Explanation-of & $\begin{array}{l}\text { Implies that the left side knowledge } \\
\text { shows the detail or gives the additional } \\
\text { explanation of the right side knowledge }\end{array}$ & $>$ & Transitivity & $\begin{array}{l}\mathrm{A}>\mathrm{B} \text { means knowledge } \mathrm{A} \text { gives } \\
\text { additional information account for } \\
\text { knowledge } \mathrm{B} \text {. We can get } \mathrm{A}>\mathrm{C} \text { if we } \\
\text { know that } \mathrm{B}>\mathrm{C} \text { is true }\end{array}$ \\
\hline Parent-of & $\begin{array}{l}\text { The existing design knowledge can be } \\
\text { packaged together in new design } \\
\text { knowledge. This relationship means the } \\
\text { right side knowledge is packaged into the } \\
\text { left side knowledge }\end{array}$ & כ & $\begin{array}{l}\text { Inverse } \\
\text { (child-of) } \\
\text { transitivity }\end{array}$ & $\begin{array}{l}\text { A } \supset \text { B means knowledge B is packaged } \\
\text { into knowledge A. And if we know B } \supset \text { C, } \\
\text { we can get A } \supset C\end{array}$ \\
\hline Dependent-of & $\begin{array}{l}\text { Some design knowledge relies on other } \\
\text { knowledge when knowledge is reused. } \\
\text { This relationship means the left side } \\
\text { knowledge relies on right side knowledge }\end{array}$ & $\ltimes$ & Transitivity & $\begin{array}{l}\mathrm{A} \ltimes \mathrm{B} \text { means knowledge } \mathrm{A} \text { is dependent } \\
\text { on knowledge } \mathrm{B} \text {. and if we know } \mathrm{B} \ltimes \mathrm{C} \text {, } \\
\text { we can get } \mathrm{A} \ltimes C\end{array}$ \\
\hline
\end{tabular}

included: favorite, sharing, comment, focus, contribute, and subscribe. Each of these represents the preference and interest of the designer to some extent. Along with the increasing number of the activities, the relationship between designer and design knowledge becomes clear gradually. And then the process of knowledge retrieval and recommendation can be improved.

The knowledge association is another very important part. The relational information between design knowledge is maintained here. In general, one design knowledge item that has more relations with others tends to easily be found out and reused. This part is precisely in order to build the relationship between design knowledge. Four relationships between design knowledge are raised, and the detail is shown in Table 2.

\section{Knowledge Reuse Patterns}

A pattern is a type of theme of recurring events or objects, sometimes referred to as elements of a set of objects. In this paper the knowledge reuse pattern (KRP) refers to the recurring ways of the designers using accumulated design knowledge. As shown in Table 3, three KRPs are identified in this paper.

4.1. Guiding Pattern. Product design is a complex problemsolving process. There are often design problems never encountered by the designer even the knowledgeable experts. For this kind of design problems, it is not enough just providing related design knowledge for the designer; a problem-solving process should also be provided to guide the designer while proper design knowledge is linked to each step involved in the process. This problem-solving process can guide the designer to decompose the current problem into subproblems and solving them one by one.

From Table 3 we can know that the linguistic and pictorial design knowledge is usually used by the guiding pattern. To support this kind of knowledge reuse pattern, the process to guide the knowledge user should be deliberately designed, 
TABle 3: Design knowledge reuse patterns.

\begin{tabular}{lcl}
\hline Reuse pattern & Knowledge types & Requirement \\
\hline Guiding & $\begin{array}{c}\text { Linguistic } \\
\text { Pictorial }\end{array}$ & $\begin{array}{l}\text { Integrate innovation methodology with design environment, provide the unified user interface to } \\
\text { guide the designer }\end{array}$ \\
\hline Reference & $\begin{array}{c}\text { Linguistic } \\
\text { Pictorial } \\
\text { Symbolic }\end{array}$ & Supply task-related knowledge and tools for design knowledge navigation \\
\hline
\end{tabular}

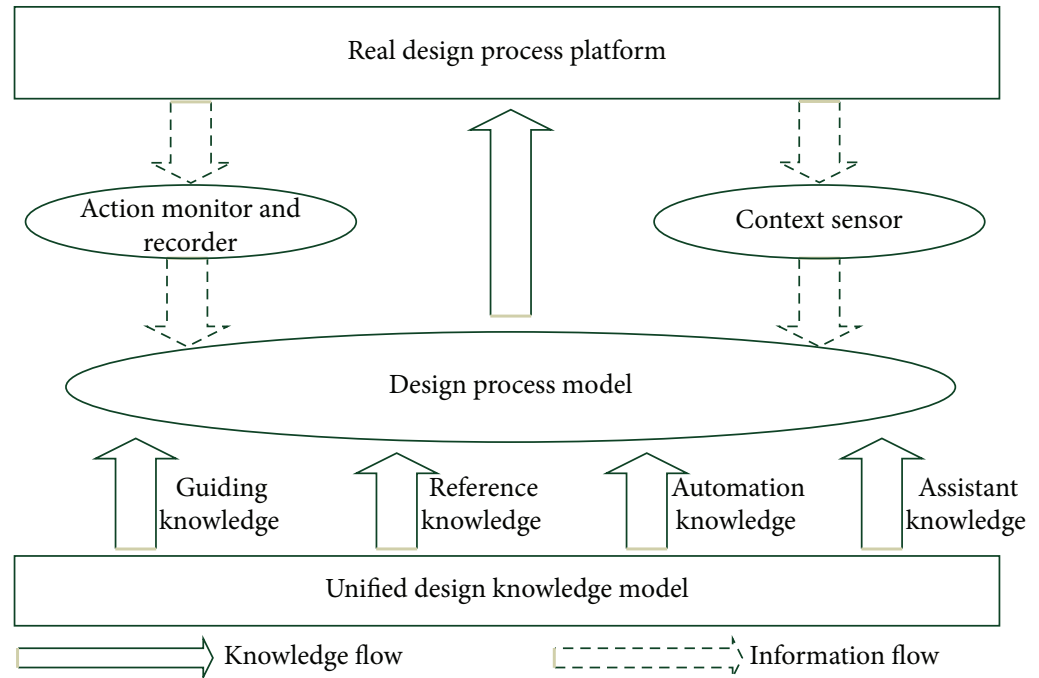

Figure 2: The design knowledge reuse model.

and the related design knowledge should be collected for each step.

4.2. Reference Pattern. As we know, there is a huge amount of design knowledge accumulated in the organizations. And much knowledge can be applied to the current design task. During the design task, designers tend to consult the related resources to make a better decision. The requirement is to browse a big amount of design knowledge and navigate among this knowledge easily.

From Table 3 we can know that all design knowledge may be used in the reference pattern. To support this kind of knowledge reuse pattern, a method to navigate user in a big amount of design knowledge is needed. We can build the relationship between design knowledge so that knowledge user can navigate to locate the design knowledge.

4.3. Automation Pattern. According to the statistics, a large portion of product design is the variant design where extensive product knowledge has been accumulated over many years, and next-generation product shares much of the same geometrical relationship. There are a huge amount of parameterized models, runnable algorithms, and repeatable processes in the product design field. Therefore, designers expect to automate the design process by utilizing the accumulated design knowledge.
From Table 3 we can know that the virtual and algorithms knowledge is usually used in the automation pattern. To support the automation pattern the key is to encapsulate a parameterized model, runnable algorithms, and repeatable processes in an opened and unified way, and then design knowledge can be reused easily and efficiently. This can be achieved by the method proposed in our previous paper [33].

\section{Design Knowledge Reuse Model}

In this paper, we propose a design knowledge reuse model which is shown in Figure 2. The most important character of the model is that design knowledge is reorganized according to the knowledge reuse patterns which are presented in the previous section. In addition, the model is designed to be changeable according to the action of the knowledge users.

As shown in Figure 2, the core element of the model is a design process model, which will be used as the framework to provide the design knowledge. The design process model is a counterpart of the real design process. The design process model will supply design knowledge to the knowledge users while the design task is carried out in the real design process. Only the basic structure of the real design process is included in the design process model, and the design knowledge is linked to each step. 


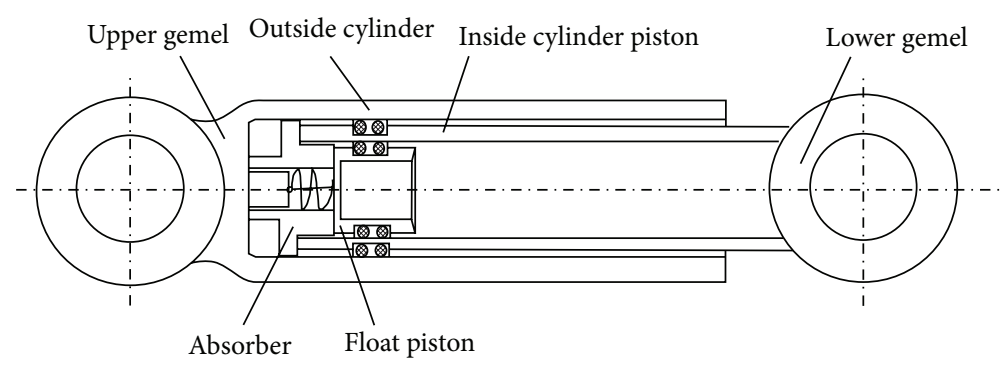

FIGURE 3: The structure of hydropneumatic spring.

During its lifecycle, the real design process platform will interact with the design process model through the action monitor/recorder and the context sensor. The context sensor is an agent for maintaining the interconnection between the design process model and the real design process. When a new task is activated in the real design process, the context sensor will catch the signal and send the task context which includes requirements, parameters, and constraints to the design process model. After that, the session will be maintained in the context sensor.

The action monitor/recorder can monitor and record the knowledge users' action silently when knowledge user browses, navigates, and executes design knowledge. The knowledge users can even put new design knowledge into the model. And these actions will be analyzed to determine what should appear in the model when the same task is activated again. By this method, the model becomes dynamic, and the design knowledge contained in the model can change continuously along with the shifty requirements of knowledge users.

Based on the unified design knowledge model, the design knowledge is reorganized according to the knowledge reuse patterns proposed in the previous section. For guiding pattern, we encapsulate design knowledge by a procedure for an engineering problem. For reference pattern, the relationship between the design knowledge is constructed for navigating the knowledge users. For automatic pattern, the KBE technology is an adaptive method, we also propose a methodology to package $3 \mathrm{D}$ models, algorithms, and design rules into an executable component in our previous work [33].

Product design is a knowledge intensive process; capturing and reuse design knowledge will benefit the design quality and cost. In the context of design knowledge reuse, this approach provides several benefits. On the one hand, this model can be used as a design knowledge organization method. Design knowledge can be organized in the same framework, and the relationship can be maintained. The ontology provides the flexibility to change the structure of design knowledge, and the reference mechanism provides the ability to handle complex semantic relationship. On the other hand, this model can be used as a middleware when developing the design knowledge system. This middleware not only can deal with the complex semantic relationship, but also can record the actions of the knowledge users and change itself according to these actions.

\section{Case Study}

We select hydropneumatic spring as the component used to illustrate the process of building knowledge reuse model. This component serves critical functions in the vehicle suspension system. Compared with other kinds of spring, the hydropneumatic spring has many advantages, like small size, light weight, and high portability.

The structure of the component is shown in Figure 3. The component constitutes of six parts including upper gemel, outside cylinder, inside cylinder piston, lower gemel, absorber, and float piston.

6.1. Knowledge and Process Identification. The design process of a product is varied at different institutes, and the underlying design knowledge is also not the same. Here we capture the design process of hydropneumatic spring from our research partner as well as the design knowledge.

As shown in Figure 4, this process can be divided into three phases. The first stage is the task definition in which the general requirements and initial parameters of the product are determined. And then the most important phase is the computed main parameter which is a subprocess. The last phase includes the geometry generation, model analysis, and report generation, in which the computed parameters in the previous phase are modeled and analyzed. In the computed main parameter phase, many steps are included, but we just list several of them in this process.

After figuring out the whole design process, we collect design knowledge for each task in the process. Taking the task which is marked by the red circle as an example, the captured design knowledge is shown in Table 4.

6.2. Knowledge Reorganization. In the previous section, the design knowledge related to each task is captured. Therefore, in this section, the collected design knowledge is reorganized according to the knowledge reuse pattern.

For the automation pattern, we have developed a method to encapsulate the symbolic, virtual, and algorithms knowledge [33]. By this method, the design knowledge can be packaged into a component which can be called through internet (web service). In this case, we packaged the input parameters, output parameters, and the algorithm by and published the function via web-service technology. For the 
TABLE 4: The design knowledge related to the suspension force analysis task.

\begin{tabular}{ll}
\hline Input & \multicolumn{1}{c}{ Activity: suspension force analysis } \\
\hline Output & Wmax: the max impact force of the tire \\
& Fsmax: the max force of the spring \\
\hline Algorithm & The empirical algorithm of computing Fss and Fsmax by Ws and Wmax (m code) \\
\hline \multirow{2}{*}{ Linguistic } & $\begin{array}{l}\text { Concept: tire's impact force, tire's max impact force, and so forth. } \\
\text { Rationale: the principle of the hydropneumatic spring, the force transmission mechanism of the vehicle, the parameter } \\
\text { computing process, and so forth. }\end{array}$ \\
& Solution: minimize the max force of the spring, and so forth. \\
Virtual & $\begin{array}{l}\text { CAD: the structure of hydro-pneumatic spring, the structure of suspension system, the structure of the chassis, and so } \\
\text { forth. } \\
\text { Animations: the animation demonstration of the principle of the hydro-pneumatic spring. }\end{array}$ \\
\hline
\end{tabular}

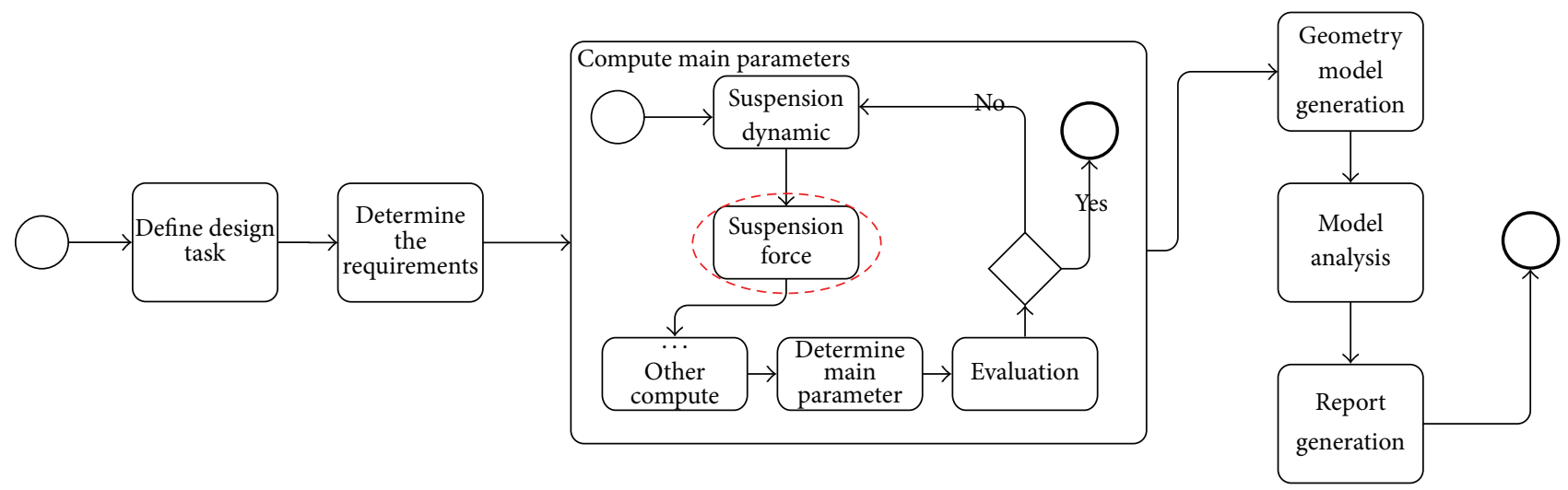

FIGURE 4: The design process of hydropneumatic spring.

\begin{tabular}{|c|c|c|}
\hline Step 1: concept & Step 2: structure & Step 3: solution \\
\hline $\begin{array}{l}\text { - Concept of FSS (knowledge } \\
\text { \#43) } \\
\text { - Concept of WS (knowledge \#42) } \\
\text { - When the max force occur } \\
\text { (knowledge \#77) } \\
\text { - The mechanical motion process } \\
\text { of the spring (knowledge \#73) }\end{array}$ & $\begin{array}{l}\text { - Structure of hydropneumatic } \\
\text { spring (knowledge \#62) } \\
\text { - The structure of suspension } \\
\text { system (knowledge \#64) } \\
\text { - The structure of the chassis } \\
\text { (knowledge \#63) }\end{array}$ & $\begin{array}{l}\text { - The number of springs } \\
\text { suspension system should affect } \\
\text { the max force } \\
\text { - The parameter of the suspension } \\
\text { offset frequency should affect } \\
\text { the max force }\end{array}$ \\
\hline
\end{tabular}

FIGURE 5: An example of the guiding knowledge.

guiding pattern, the collected design knowledge is packaged in a step-by-step form. The knowledge user can locate the knowledge following the process. Figure 5 shows the guiding process of the knowledge: "minimize the max force of the spring." For the reference pattern, the relationships between design knowledge are constructed as stated in Section 3.

6.3. Ontology Based Knowledge Reuse Model. In this section, we use ontology to represent the knowledge reuse model because of the flexibility of the ontology. And we build the ontology model with protege which is a free, open source ontology editor. First, the metamodel is constructed in protege environment. The basic content of the metamodel is shown as Figure 6. Four object properties, including containaction, done-by, have-knowledge, and with-knowledge, construct the main structure of the metamodel. The containaction records which actions occurred in a specific task. The done-by means who performs a specific action. The have-knowledge means which knowledge will be provided to a specific task. There are four subproperties including have-assistant, have-automation, have-guiding, and havereference. The first is used to provide domain expert information, and the other three are used to prove the collected knowledge. The range and domain of these object properties are indicated by blue and red lines.

Based on this metamodel, we instantiate the hydropneumatic spring example. All collected design knowledge 


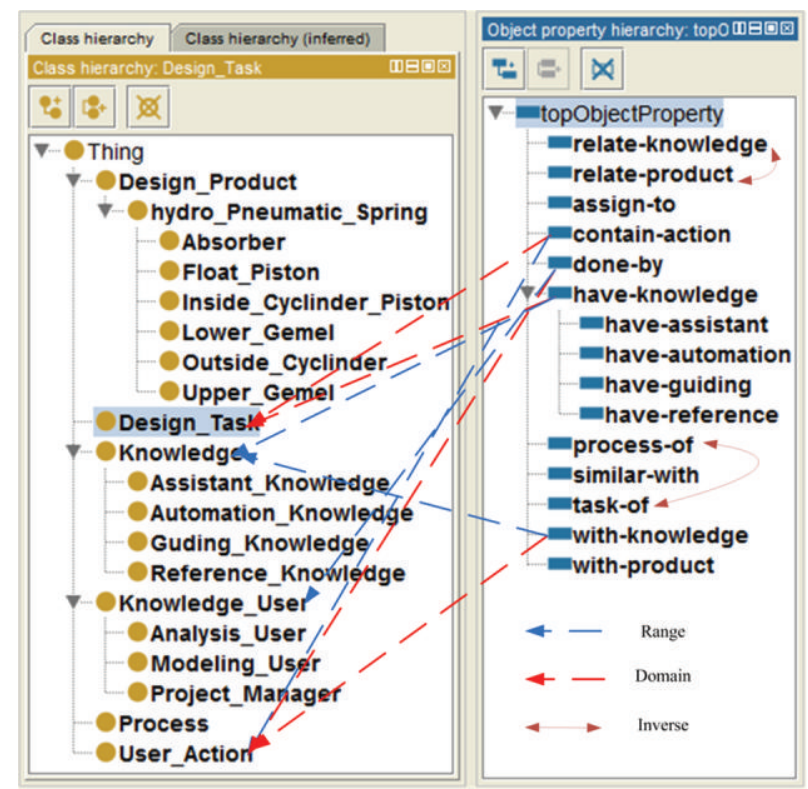

FIGURE 6: The basic classes and object property of the knowledge reuse model.

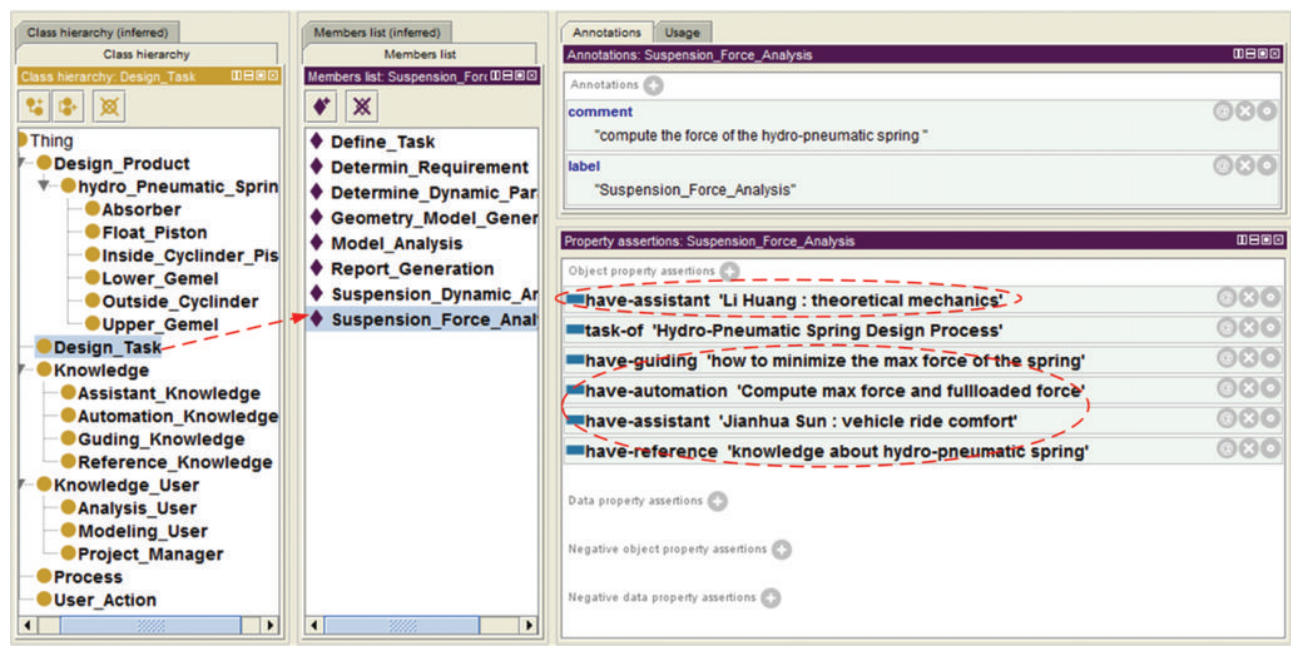

FIGURE 7: The detail information of Suspension_Force_Analysis task.

is represented as individuals of knowledge. Figure 7 shows the detail information of Design_Task labeled Suspension_Force_Analysis. All tasks of the hydropneumatic spring design process are defined as the individuals. Each individual of Design_Task is assigned to an individual of process. The assertions marked by the red circles are to build the connection between Design_Task and knowledge; it implies that these declared individuals of knowledge will be provided to knowledge user.

Assuming one action has been captured during Suspension_Force_Analysis task, the model provides the ability to update itself. After the User_Action is captured, it will be listed in the object property assertions area as shown in Figure 8.
We use FaCT++ (http://owl.man.ac.uk/factplusplus/) to infer the model. As you can see, we get another five assertions, four of them can be inferred directly as the blue dashed lines indicate while the last one needs a detailed explanation. In fact, the last inferred assertion originates in the containaction activity 1 assertion. On the one hand, this assertion means that the design task includes an activity, and the upper right corner of Figure 8 shows that activity 1 deals with the knowledge "Jianhua Sun: vehicle ride comfort." On the other hand, when we declare the object property of haveknowledge, a property chain is defined in Algorithm 1.

The owl code means that, if $\mathrm{T}$ contain-action $\mathrm{A}$ and $\mathrm{A}$ with-knowledge $\mathrm{K}$, then we have T have-knowledge $\mathrm{K}$. So this 

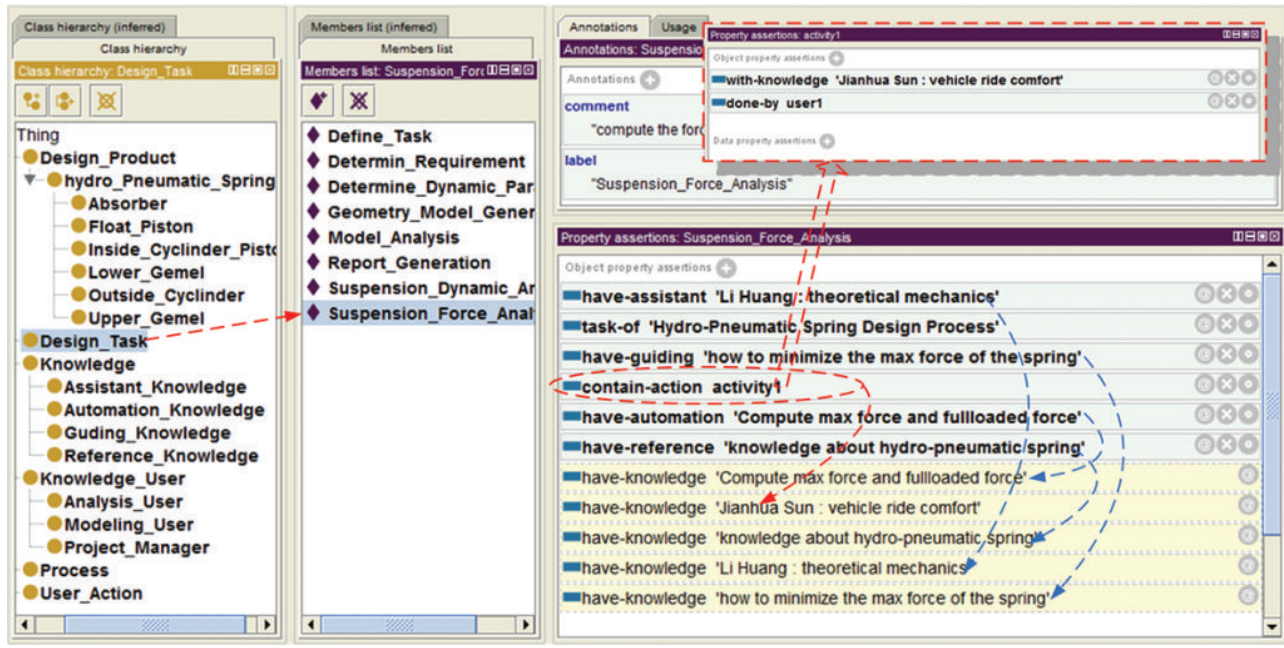

Figure 8: Inference result of the Suspension_Force_Analysis task.

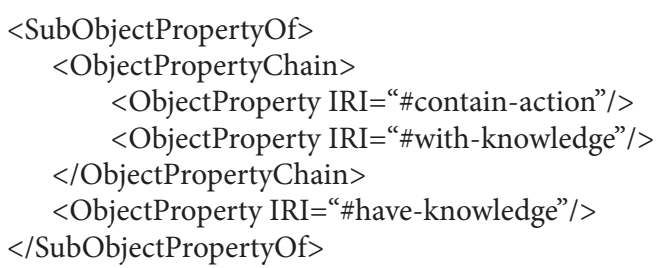

Algorithm 1

is why we can reason the knowledge "Jianhua Sun: vehicle ride comfort" should be provided to the Knowledge_User.

\section{Conclusion}

Aiming to ease the design knowledge reuse, we first discussed the design knowledge and proposed a unified design knowledge model. Following that, three knowledge reuse patterns of the knowledge user are identified. Based on that, we developed a knowledge reuse model which takes the knowledge user into account. On the one hand, the knowledge reuse model organizes the design knowledge according to the identified knowledge reuse pattern. On the other hand, the model can capture the actions of the user and update itself according to these actions. This work attempts adding the users' requirements into the knowledge reuse model and makes the model easier to be accepted by knowledge users.

Despite the progress of the method, there are some issues that needed to be addressed by future work. First, the design process can be divided into several stages. Which knowledge reuse patterns fit for a specific stage of the design process is needed to be studied. Second, the model should be used to develop a design knowledge management system to validate whether the design knowledge provided by the model can satisfy the requirements of the knowledge user.

\section{References}

[1] A. A. Ammar, D. Scaravetti, and J. P. Nadeau, "Knowledge reuse: Towards a design tool," in Proceedings of the 11th International Design Conference (DESIGN '10), pp. 1451-1460, Dubrovnik, Croatia, May 2010.

[2] Y. S. Kim and K. Y. Kim, "DCR-based causal design knowledge evaluation method and system for future CAD applications," CAD Computer Aided Design, vol. 44, pp. 947-960, 2012.

[3] N. Reed, J. Scanlan, G. Wills, and S. T. Halliday, "Knowledge use in an advanced manufacturing environment," Design Studies, vol. 32, no. 3, pp. 292-312, 2011.

[4] K. Kim and Y. S. Kim, "Causal design knowledge: alternative representation method for product development knowledge management," CAD Computer Aided Design, vol. 43, no. 9, pp. 1137-1153, 2011.

[5] N. Iyer, S. Jayanti, K. Lou, Y. Kalyanaraman, and K. Ramani, "Shape-based searching for product lifecycle applications," CAD Computer Aided Design, vol. 37, no. 13, pp. 1435-1446, 2005.

[6] D. Baxter, J. Gao, K. Case et al., "An engineering design knowledge reuse methodology using process modelling," Research in Engineering Design, vol. 18, no. 1, pp. 37-48, 2007.

[7] J. Bryson, J. J. Cox, and J. T. Carson, "A product development scenario for knowledge capture and reuse," Computer-Aided Design and Applications, vol. 6, no. 2, pp. 207-218, 2009.

[8] D. Baxter, J. Gao, K. Case et al., "A framework to integrate design knowledge reuse and requirements management in engineering 
design," Robotics and Computer-Integrated Manufacturing, vol. 24, no. 4, pp. 585-593, 2008.

[9] W. J. C. Verhagen, P. Bermell-Garcia, R. E. C. Van Dijk, and R. Curran, "A critical review of knowledge-based engineering: an identification of research challenges," Advanced Engineering Informatics, vol. 26, no. 1, pp. 5-15, 2012.

[10] G. L. Rocca, "Knowledge based engineering: between AI and CAD. Review of a language based technology to support engineering design," Advanced Engineering Informatics, vol. 26, no. 2, pp. 159-179, 2012.

[11] K. Amadori, M. Tarkian, J. Ölvander, and P. Krus, "Flexible and robust CAD models for design automation," Advanced Engineering Informatics, vol. 26, no. 2, pp. 180-195, 2012.

[12] P. Bermell-Garcia, W. J. C. Verhagen, S. Astwood et al., "A framework for management of knowledge-based engineering applications as software services: enabling personalization and codification," Advanced Engineering Informatics, vol. 26, no. 2, pp. 219-230, 2012.

[13] M. J. Foeken, A. A. Alvarez Cabrera, M. Voskuijl, and M. J. L. Van Tooren, "Enabling control software generation by using mechatronics modeling primitives," Advanced Engineering Informatics, vol. 26, no. 2, pp. 196-206, 2012.

[14] D. Steenhuizen and M. Van Tooren, "The implementation of a knowledge-based framework for the aerodynamic optimization of a morphing wing device," Advanced Engineering Informatics, vol. 26, no. 2, pp. 207-218, 2012.

[15] C. Van Der Velden, C. Bil, and X. Xu, "Adaptable methodology for automation application development," Advanced Engineering Informatics, vol. 26, no. 2, pp. 231-250, 2012.

[16] H. Wang, A. L. Johnson, and R. H. Bracewell, "The retrieval of structured design rationale for the re-use of design knowledge with an integrated representation," Advanced Engineering Informatics, vol. 26, no. 2, pp. 251-266, 2012.

[17] L. Jia, J. Hong, Z. Qiu, B. Li, H. Yan, and S. Pan, "Initialized design knowledge model constructing for complex structural components," Journal of Xian Jiaotong University, vol. 42, no. 7, pp. 865-869, 2008.

[18] I. P. Tama and C. Reidsema, "Product knowledge identification and modeling for virtual collaboration environment," in Proceedings of the Portland International Center for Management of Engineering and Technology-Technology Management for Global Economic Growth (PICMET '10), pp. 1029-1037, Phuket, Thailand, July 2010.

[19] D. Baxter, R. Roy, A. Doultsinou, J. Gao, and M. Kalta, "A knowledge management framework to support product-service systems design," International Journal of Computer Integrated Manufacturing, vol. 22, no. 12, pp. 1173-1188, 2009.

[20] G. Feng and D. Cui, "Research of design reuse in complex product integrated design systems," in Proceedings of the IEEE International Conference on Intelligent Computing and Intelligent Systems (ICIS '10), pp. 750-754, Xiamen, China, October 2010.

[21] Y. Li, X. Shao, P. Li, and Q. Liu, "Design and implementation of a process-oriented intelligent collaborative product design system," Computers in Industry, vol. 53, no. 2, pp. 205-229, 2004.

[22] Y. Li, W. Zhao, and L. Hu, "A Process oriented hybrid resource integration framework for product variant design," Journal of Computing and Information Science in Engineering, vol. 12, no. 4, Article ID 041005, 14 pages, 2012.

[23] C. M. Lu, S. Zheng, W. Jin, Y. F. Sheng, and Y. Sun, "Templatebased expression and reuse of design knowledge," Advanced Materials Research, vol. 328-330, pp. 274-278, 2011.
[24] F. Montagna, "Decision-aiding tools in innovative product development contexts," Research in Engineering Design, vol. 22, no. 2, pp. 63-86, 2011.

[25] P. P. Kumar, Design Process Modeling: Towards an Ontology of Engineering Design Activities, 2008.

[26] S. K. Sim and A. H. B. Duffy, "Towards an ontology of generic engineering design activities," Research in Engineering Design, vol. 14, no. 4, pp. 200-223, 2003.

[27] X. Liu, D. Rosen, and Z. Yu, "Ontology based knowledge modeling and reuse approach in product redesign," in Proceedings of the 11th IEEE International Conference on Information Reuse and Integration (IRI '10), pp. 270-273, Las Vegas, Nev, USA, August 2010.

[28] Z. Yu, "Ontology based knowledge management and reuse approach in digital design," in Proceedings of the 1st IEEE International Conference on Progress in Informatics and Computing (PIC '10), pp. 360-363, Shanghai, China, December 2010.

[29] B. Song and Z. Jiang, "Proactive search enabled contextsensitive knowledge supply situated in computer-aided engineering," Advanced Engineering Informatics, vol. 27, pp. 66-75, 2013.

[30] J. E. Ettlie and M. Kubarek, "Design reuse in manufacturing and services," Journal of Product Innovation Management, vol. 25, no. 5, pp. 457-472, 2008.

[31] R. Owen and I. Horvath, "Towards product-related knowledge asset warehousing in enterprises," in Proceedings of the 4th international symposium on tools and methods of competitive engineering (TMCE '02), pp. 155-170, Hubei, China, 2002.

[32] S. K. Chandrasegaran, K. Ramani, R. D. Sriram et al., "The evolution, challenges, and future of knowledge representation in product design systems," Computer-Aided Design, vol. 45, pp. 204-228, 2013.

[33] J. Hao, H. C. Yang, Y. Yan, and G. X. Wang, "Configurable knowledge component technology oriented to product design tasks," Computer Integrated Manufacturing Systems, vol. 18, pp. 705-712, 2012. 

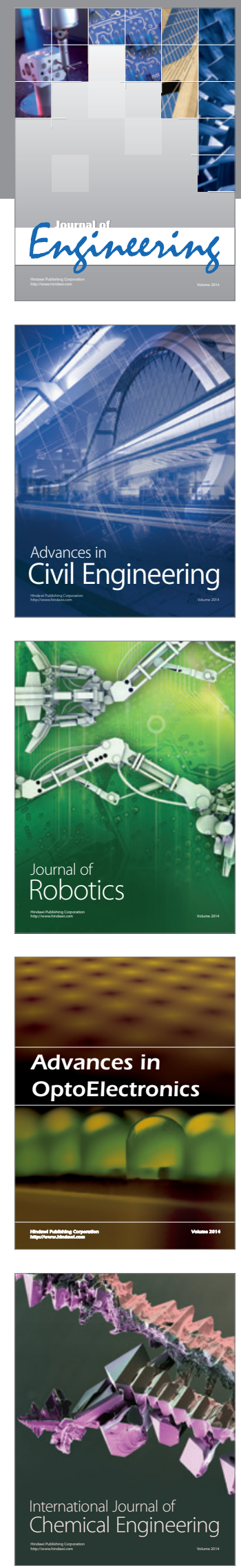

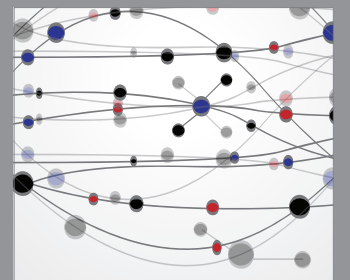

The Scientific World Journal
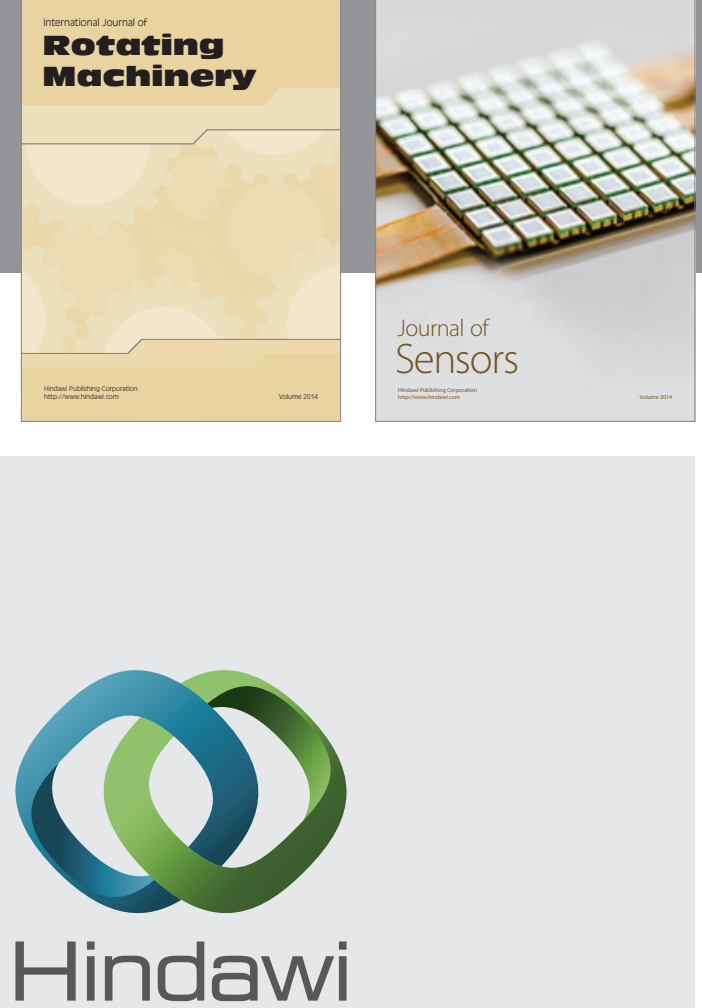

Submit your manuscripts at http://www.hindawi.com
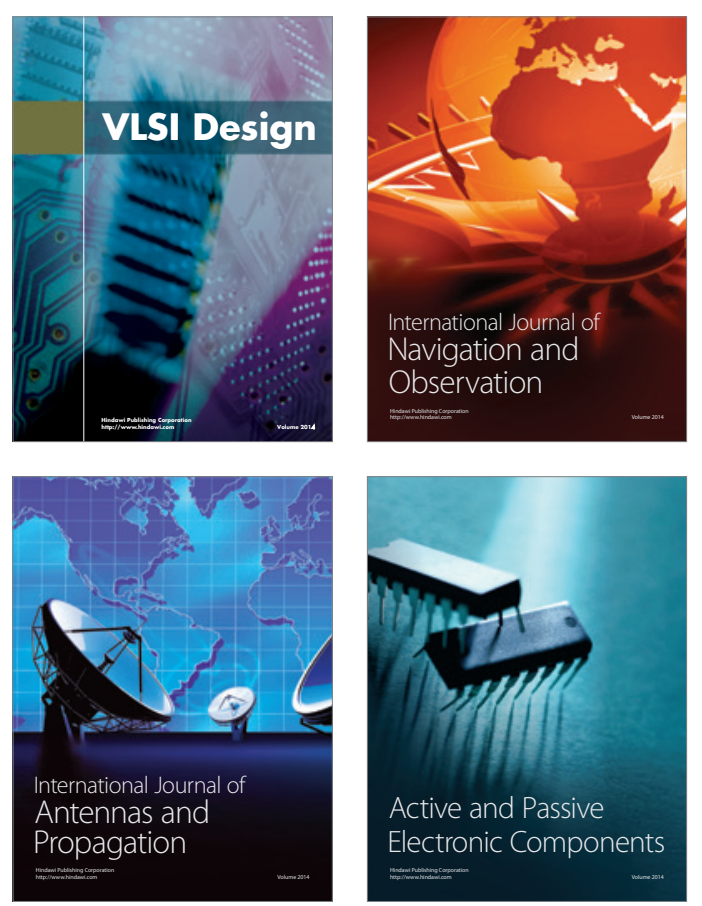
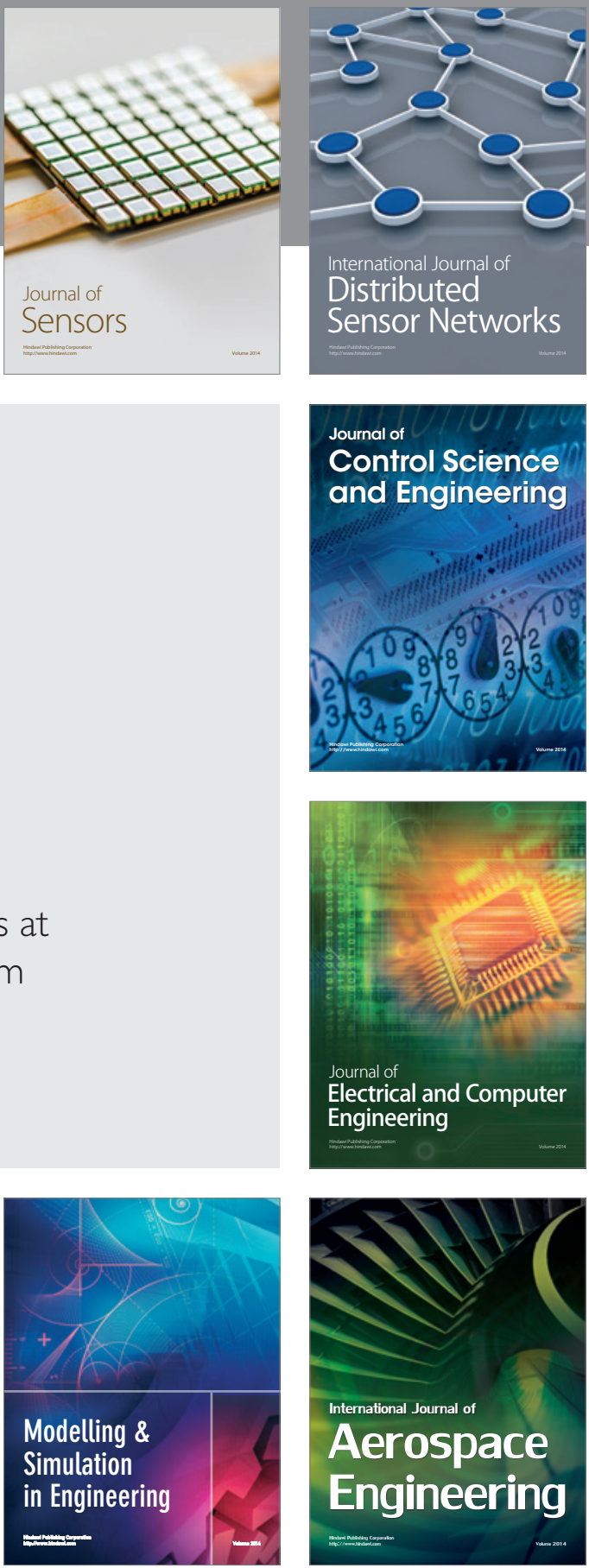

Journal of

Control Science

and Engineering
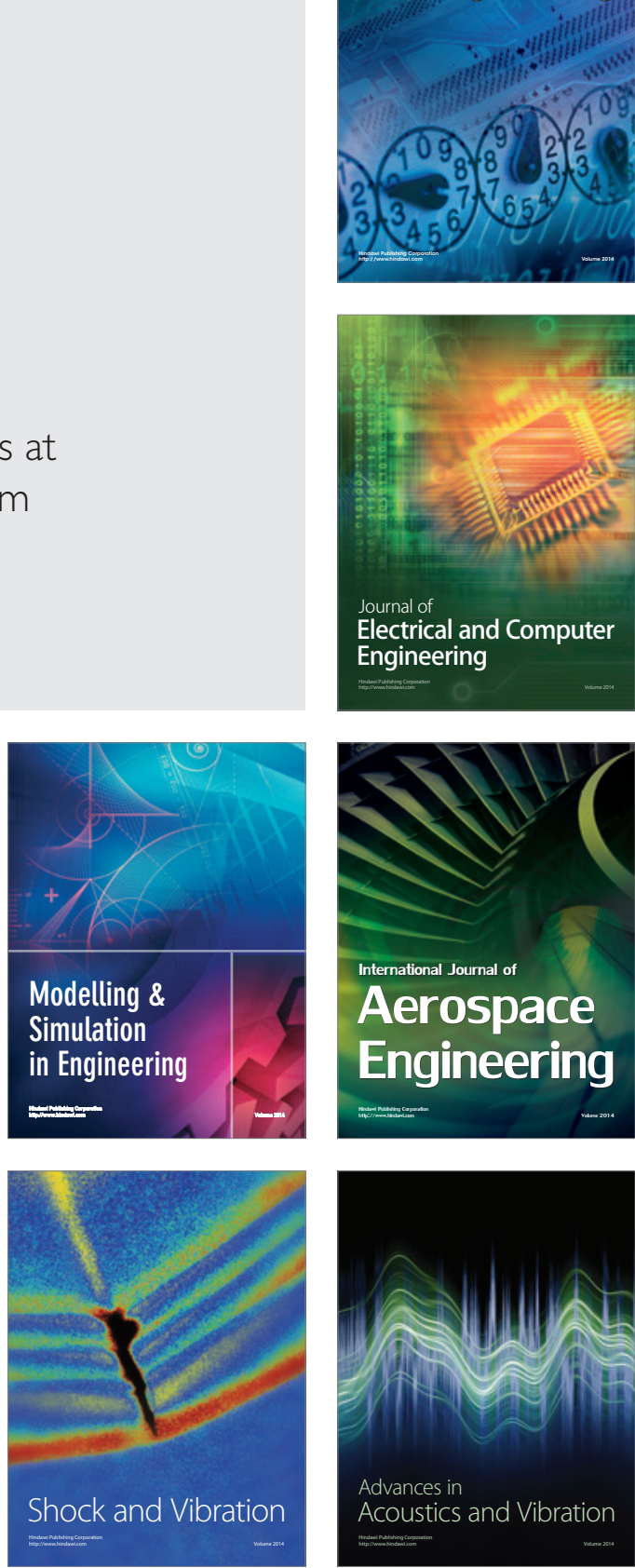